\title{
Modified Method of Texture Feature Extraction and Analysis using Daubechies Wavelet and SVM
}

\author{
Debasish Deb \\ M.Tech Scholar \\ National Institute Of Technology \\ Agartala, India
}

\author{
Parthasarathi De \\ Asstt. Professor \\ National Institute Of Technology \\ Agartala, India
}

\author{
Nikhil Debbarma \\ Asstt. Professor \\ National Institute Of Technology \\ Agartala, India
}

\begin{abstract}
Text Extraction is always a challenging problem in the field of research area. In this paper we developed an algorithm that can be used to extract text from images. We have studied texture feature analysis and also successfully retrieve the texture features embedded in an image. We also calculated number of characters in blurred images. Our algorithm is successfully implemented on many images that are collected from ICDAR Dataset 2011, ICDAR 2013, ICDAR 2003 and some images are collected from newspapers, and digital camera. We used different methods for texture feature extraction. Our algorithm gives more efficiency and extract texture features as compared with previous studies.
\end{abstract}

\section{General Terms:}

Texture Feature extraction, Pattern Recognition

\section{Keywords:}

Preprocessing,Daubechies Wavelet,SVM,Otsu Method

\section{INTRODUCTION}

The goal of this project is to extract text from images using different approaches like edge-based, connected component based, wavelet based along with some other methods such as application of Otsu method, Support Vector Machine, Morphological Operation, Segmentation technique, Wavelet transformation techniques. Text embedded in an image contain some information in the form of text, our aim is to extract those text from images and also calculation of number of character in text that are embedded in a blurred images. For our experiment purposes we used ICDAR 2013 and ICDAR 2011 dataset, and ICDAR 2003 dataset. We also compared our experiment results with previous years published papers and we have fond that our methods gives efficient results as compared with the data set that are used for text extraction and mentioned in previous papers. In the field of digital image processing the study of texture feature extraction is difficult; it is a big and challenging problem. Extraction of text from images is difficult if the images contains blurred texts or if the text contains background complexity or low resolution, then it will be difficult to extract text and calculate the number of character in the blurred images. In our study we have worked on images that contain text that is mixed with background.
We successfully extracted text and also calculated characters that are mixed with background. Printed text on magazine and book covers are mostly embedded with pictures. Semantic and useful information [1] may be collected from images, so we need to separate text strings from images. Various degradations such as blurriness, low resolution, or uneven lightening, make it difficult to extract text from images having noise. In this paper, we proposed a hybrid method for extraction of text that is embedded in images. Text in an image contain varies properties such as color, edge, size [2]. Figure below shows the schematic representation of our implementation in text extraction.

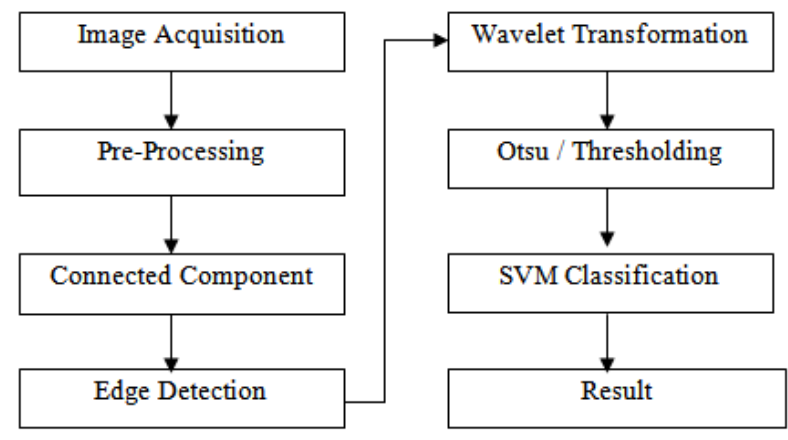

Fig.1. Implementation of Text Extraction methods

\section{TEXT EXTRACTION : A SURVEY}

Texture feature analysis, text extraction and detection in images are difficult problem in the field of digital image processing research area [3]. We did a lot of research and highlighted previous 10 years papers. We have worked on these fields to get a good result. Canny edged detector is basically used to get possible text regions but Sobel edge detector cannot detect possible text regions and the connected component analysis is applied to get the candidate text regions. If image contain background complexity then possibly non text regions also get collected [4], so we need to remove non text regions by texture feature analysis. Text extraction method is based on K-means clustering in HSI color space, Euclidian distance in RGB color space; Euclidian distance provides good results that represent color difference measure [5]. Another method that is used in text detection is connected component that considers the texts in images as a set of connected components and possess particular ge- 
ometric characteristics which are closely connected to the space of the surrounding components and its geometric characteristics [8]. Text recognition was mentioned by a text segmentation step which was followed by OCR algorithm, statistics and language modeling. Sunil Kumar et al. [6] proposed a clustering-based technique for estimating globally matched wavelet filters using a collection of ground truth images. Fisher classifiers have been used for classification. S.Audithan and RM. Chandrasekaran [9] proposed that Haar discrete wavelet transform operation was fastest among all wavelets. 1 or -1 represents Haar coefficients. Detection of edges, line feature vector graph was generated based on the edge map and the stroke information was extracted. Finally the regions that contain text was generated and filtered based on line features. Emanuel Indermuhle et al. [5] introduced two systems.

\section{METHODOLOGY}

The project work is implemented on MATLAB2013a version. For our experiment purposes we used ICDAR 2013 and ICDAR 2011 dataset, and ICDAR 2003 dataset. We used different approaches to extract text from images. First the image is taken from dataset and some collected from camera. The taken images is pre-processed, it undergoes various degradation such as low resolutions, blurriness, lightening. Per-processing is a technique that involves in removing low-frequency background noise, adjusting size of images, normalizing the intensity of the individual particles images, removing reflections, and masking portions of images. We need preprocessing technique to enhance dataset images prior to computational processing. Next step is to convert original rgb images into grayscale images. We applied Canny and Sobel edge detection technique to get the possible text area regions. It is always good to apply canny detection technique rather than Sobel edge detection technique; Median is used to remove noises, Gradient Difference gives text regions of high contrast, Connected Component analysis gives connectivity, a way of dimension using 3 by 3 matrix, it consists of 0's and 1's; Which is always symmetric about its center, it is based on spatial coordinates. Next we applied Daubechies DWT that gives characteristics of textured image, Otsu Method used to remove non-text regions and at last SVM is used for classification. Image segmentation is the process of dividing an image into number of constituent part so that each part can be processed individually. The purpose is to subdivide an image into meaningful nonoverlapping regions, which would be used for further analysis. It is hoped that the regions obtained correspond to the physical parts or objects of a scene (3-D) represented by the image (2-D). We used SVM to classify text regions from non-text regions. SVM plays an important role for separating text from non text regions.

\section{EXPERIMENTS AND RESULTS}

The project work is implemented on MATLAB2013a version. For our experiment purposes we used ICDAR 2013 and ICDAR 2011 dataset, and ICDAR 2003 dataset. We also compared our experiment results with previous years published papers and we have fond that our methods gives best and most efficient results as compared with the data set that are used for text extraction and mentioned in previous papers. For the sake of simplicity we used two images as shown in figure 2 below. At last we used SVM; this is a kind of large-margin classifier, SVM is used for classification, it is a space vector based machine learning method, SVM is used to find a decision boundary between two classes that is maximally far from any point in the training data. Basically there is no accepted method that can be used to evaluate the efficiency of the extraction method and text localization, we use several metrics used by others to facilitate a meaningful comparison. In our proposed method, we compute the accuracy of the algorithm output by manually counting the number of correctly located characters. We also calculated Precision rate and recall rate. We have tested these images and found that our method is giving above $95 \%$ accuracy. Intensity of an image $\mathrm{Y}$ is given as:

$$
\mathrm{Y}=\mathrm{R}(.29)+\mathrm{G}(.58)+\mathrm{B}(.11)
$$

For our experiment we used these picture and extracted text successfully. Figure below shows the original picture. One is text with background and other is blurred.

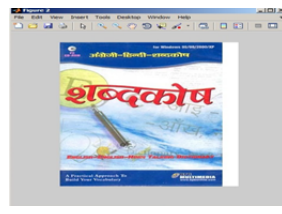

Fig. 2. Text with complex background

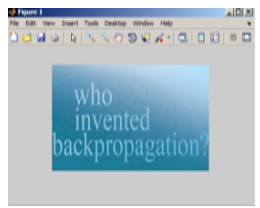

Fig.3. Text with blurred background

After the images have been successfully preprocessed and undergone various implementation phase we have got the result as shown below

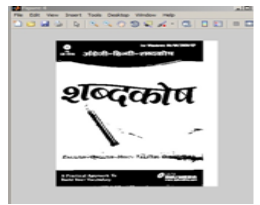

Fig. 2(a) Extracted text from image with background

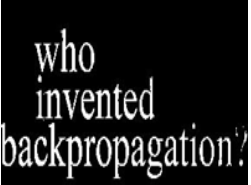

Fig. 3(b) Extracted text from blurred image

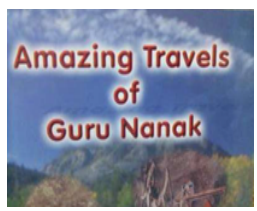

Fig. 4(a) Original image taken from ICDAR Dataset

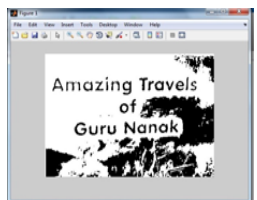

Fig. 4(b) Extracted text from from complex background

The text which is embedded in image is also extracted and the number of character is also calculated by using the methods below: 
img1=imread('imageprocessing.jpg');

imshow (img1)

img1=rgb2gray(img1);

imshow(img1) img2=im2bw(img1, graythresh(imageprocessing));

imshow(img2)

img2 $=$ img2;

imshow(img2)

$\mathrm{B}=$ bwboundaries(img2);

imshow (img2)

text $(10,10$,strcat $(' \backslash$ color $\{$ green $\}$ Objects

Found:',num2str(length(B))))

hold on

for $\mathrm{k}=1$ : length $(\mathrm{B})$

boundary $=\mathrm{Bk}$;

plot (boundary (:, 2), boundary(:,1), 'g', 'LineWidth', 0.2)

end

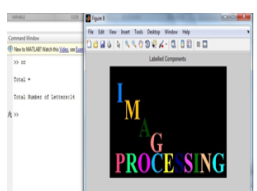

Fig. 5. Calculation of number of character in image

Table below shows the extraction of text from images and that are collected from camera and some are collected from standard database and the results of experiment conducted in system laboratory.

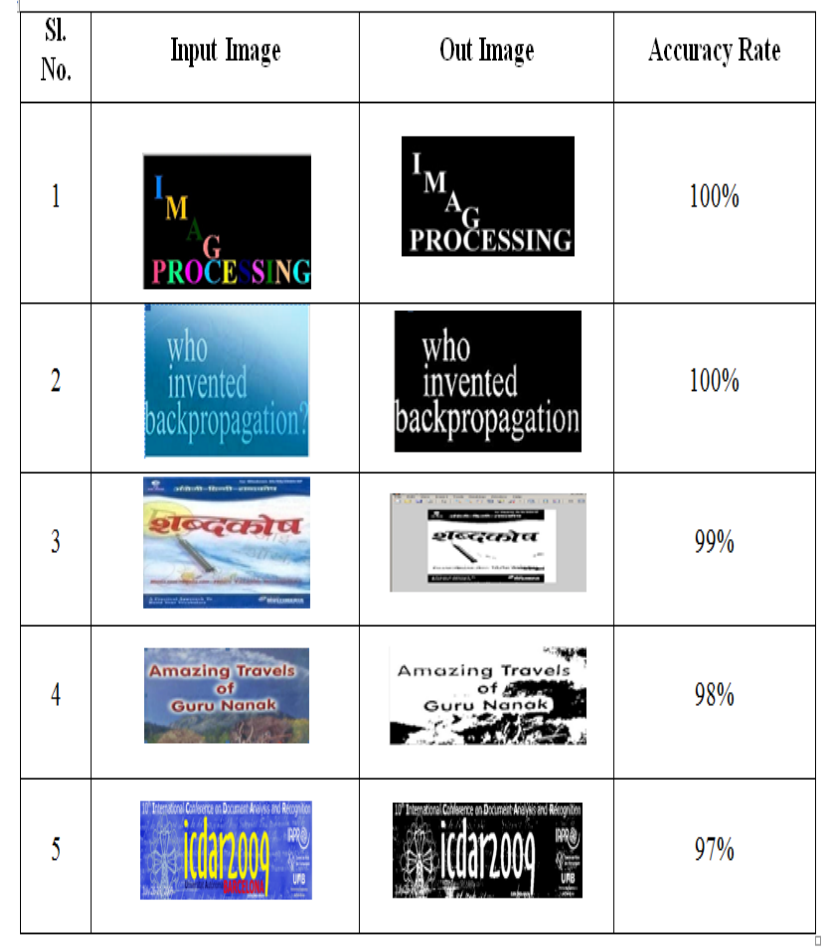

Fig. 6. Table showing the accuracy rate of the extracted text

\section{NOISE REMOVAL AND IMAGE ENHANCEMENT}

We use median filter to remove noise. Adaptive Median Filtering and Wiener 2 is used to perform more smoothing of image. It is better to use adaptive filter than linear filter because it also preserves edges and other high-frequency parts of an image.

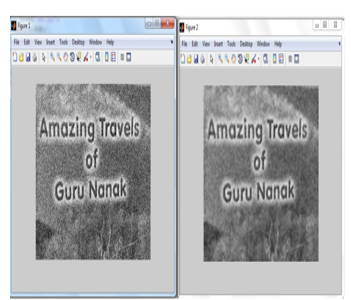

Fig.7. Adaptive median filter for removing noise

Image enhancement is the process that adjusts digital images so that the results are more suitable for display or further image analysis. Figure below shows the Image Enhancement process. Using histogram equalization technique the contrast of image can be enhanced. Histogram equalization distributes pixel values throughout a range, thus, equalizing and making the histogram more "normal" shaped. The disadvantage of this approach is that it can enhance noise as well. Histogram equalization improves contrast but not sharpness or other quality metrics. Many of the images have varying intensity level (read different values of pixel data), which sometimes makes data (read: objects in images) in the foreground and background indistinguishable (not clearly visible). The basic equation of image histogram is given below

$$
\mathrm{J}=\text { histeq (I) }
$$

Figure below shows the histogram equalization technique of the contrast of image it can also be enhanced

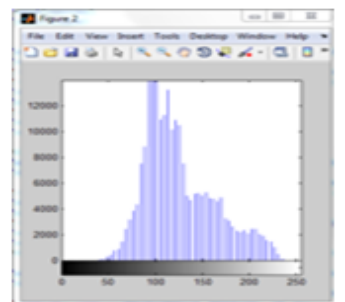

Fig. 8 Histogram equalization technique of original image

Image is enhanced after being processed of enhancement function of basic MATLAB function

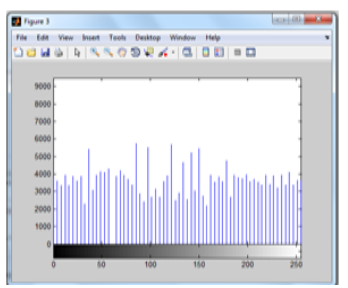

Fig. 9 Histogram of image after being enhanced

\section{PRECISION AND ACCURACY RATE}

The results obtained now are more efficient as compared with the previous results. The experiment gives above $90 \%$ precision and 
accuracy rate. We have also calculated the False Negative, False Positive and Recall rates and found efficient results.

(1) False Negatives (FN): It means it will miss those regions which are actually text characters, but not detected by the algorithm.

(2) False Positives (FP): False alarms represent those regions which are not characters of a text, but detected by the algorithm as text.

(3) Recall Rate (r): Ratio of the correctly detected characters to sum of correctly detected characters plus false negatives.

$$
\text { Recall Rate }=\frac{\text { correctly detected characters }}{\text { correctly detected characters }+\mathrm{FN}}
$$

(4) Precision Rate (p): represents ratio of correctly detected characters to the sum of correctly detected characters plus false positives. Mathematically we can represent it by a formula as shown below

$$
\text { Precision Rate }=\frac{\text { correctly detected characters }}{\text { correctly detected characters }+\mathrm{FP}}
$$

(5) F-score represents harmonic mean (HM) of the recall rate and precision rates.

$$
\mathrm{F} \text {-score }=\frac{\text { precision } . \text { Recall }}{\text { Precision }+ \text { Recall }}
$$

(6) Accuracy Rate: The accuracy of a measurement is how close a result comes to the true value.

$$
\text { Accuracy Rate }=\frac{\mathrm{TP}+\mathrm{TN}}{\mathrm{TP}+\mathrm{TN}+\mathrm{FP}+\mathrm{FN}}
$$

Where $\mathrm{TP}=$ Total Positive and $\mathrm{TN}=$ Total Negative

We have calculated the precision rate and accuracy rate of the tested images. Table below shows the results Evaluation performance, Precision Rate, Recall Rate and Accuracy Rate of our experiments of the tested images

\begin{tabular}{|c|c|c|c|c|}
\hline PARAMETER & PRECTSION RATE & RECALL RATE & F-SCORE & ACCURACY RATE \\
\hline $\begin{array}{c}\text { Proposed } \\
\text { Method }\end{array}$ & 97.65 & 96.69 & 97.17 & $98.8 \%$ \\
\hline
\end{tabular}

Fig.10 Evaluation performance of tested images

\section{FUTURE WORK}

We are still working to extract text for hidden area region; text extraction in a cluttered scene, more work should be done on wavelet. We are working for implementation of CWT operations, filtering Operations More work should be done on background complexity; automatic cropping of the portion of text embedded in images; rational devices that guide the reader through; We are trying to implement new method that will give the text string embedded in an images. We have calculated the number of characters in the text, embedded in an image.

\section{CONCLUSION}

The Text Extraction in an image is more and challenging and interesting in research area. We have successfully extracted text embedded in the images, but still there are some issues, we have to work for future we have done the initial phase of the system like, image acquisition, conversion to different types and recognition of characters in the images.Our future work will be based on CWT, and we will try to analyze texture feature for the images with complex background.

\section{REFERENCES}

[1] Niti Syal1, Naresh Kumar Garg, "Text Extraction in Images Using DWT, Gradient Method And SVM Classifier?, International Journal of Emerging Technology and Advanced Engineering. Volume 4, Issue 6, June 2014

[2] A.J.Jadhav, Vaibhav Kolhe and Sagar Peshwe, "Text Extraction from Images: A Survey", International Journal of Advanced Research in Computer Science and Software Engineering., Vol. 3, Issue 3, March 2013

[3] Sneha Sharma, "Extraction of Text Regions in Natural Image", Department of Computer Science, Rochester Institute of Technology,Spring 2006/07

[4] P.Nagabhushan, S.Nirmala "Text Extraction in Complex Color Images for Enhanced Readiability ," http://www.scirp.org/journal/iim.

[5] Matko, Hrvoje ,Mladen, "Scene text extraction in HIS color space using K-Means and modified cylindrical distance" in PRZEGLAD ELEKTROTECHNICZNY .pp 117, Year 2013

[6] [6] Sunil Kumar, Rajat Gupta, Nitin Khanna, Santanu Chaudhury, and Shiv Dutt Joshi, "Text Extraction and Document Image Segmentation Using Matched Wavelets and MRF Model,” IEEE Trans. On image processing, Vol. 16, No. 8.

[7] S.Audithan and RM. Chandrasekaran, "Document Text Extraction from Document Images Using Haar Discrete Wavelet Transform, "European Journal of Scientific Research, ISSN 1450-216X ,Vol.36, No.4, pp.502-512,2009.

[8] Emanuel Indermhle, Horst Bunke, Faisal Shafait and Thomas Breuel, “ Text versus non-Text Distinction in Online Handwritten Documents," ACM, 978-1-60558-638-0/10/03, pp.22?26, 2010.

[9] Wenhua Dai, Cuizhen Jiao, Tingting He; Research on Text Feature Extraction Based on Hybrid Parallel Genetic Algorithm; Wireless Communications, Networking and Mobile Computing, 2007. WiCom 2007. International Conference on DOI: 10.1109/WICOM.2007.1367 Publication Year: 2007 , Page(s): 5581 - 5584

[10] Rong-Chi Chang, "Intelligent Text Detection and Extraction from Natural Scene Images", Nano, Information Technology and Reliability (NASNIT), 2011, Publication Year: 2011, Page(s): 23- 28.

[11] Rong-Chi Chang, Intelligent Text Detection and Extraction from Natural Scene Images; Nano, Information Technology and Reliability (NASNIT), 2011 15th North-East Asia Symposium on DOI: 10.1109/NASNIT.2011.6111115 ; Year: 2011, Page(s): 23-28

[12] Yang Zhang ; Inst. of Autom., Beijing, China ; Chunheng Wang ; Baihua Xiao ; Cunzhao Shi,"A New Text Extraction Method Incorporating Local Information";IEEE International Conference on Frontiers in Handwriting Recognition (ICFHR), 2012; Year:2012, pp.252 - 255

[13] Hongwei Zhang ; Changsong Liu ; Cheng Yang ; Xiaoqing Ding, "An Improved Scene Text Extraction Method Using Conditional Random Field and Optical Character Recognition", IEEE International Conference on Document Analysis and Recognition (ICDAR), Year 2011, Page(s):708 - 712.

[14] Peng Tianqiang, Tian Pohuang, Li Bicheng, "A robust video text extraction method based on text traversing line and stroke connectivity", IEEE 9th International Conference on Signal Processing, 2008. ICSP 2008. Page(s): 1002 - 1005 